\title{
UNA MIRADA A LA INCLUSIÓN COMO OPORTUNIDAD DE DESARROLLO
}

\author{
AUTORES: $\quad$ Lidia Esther García Grillo ${ }^{1}$ \\ Yisell Vigoa Escobedo ${ }^{2}$ \\ Dayamí Álvarez Ayala ${ }^{3}$ \\ Hilda Brigitte Quijije Quiroz ${ }^{4}$
}

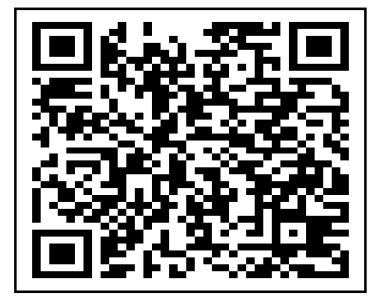

\section{DIRECCIÓN PARA CORRESPONDENCIA: (Igarciagrillo@udg.co.cu)}

Fecha de recepción: 12/04/2021

Fecha de aceptación: 22/09/2021

\section{RESUMEN}

La investigación aborda experiencias sobre la atención a las personas con necesidades educativas especiales, asociadas o no a discapacidades, desde la relación que se establece entre los contextos escolar, familiar y comunitario. Se desarrolla en el municipio Bayamo a través de talleres de socialización e involucramiento, en los que se considera como elementos esenciales, la retroalimentación que debe existir entre la escuela, la familia y la comunidad como agentes potenciadores del desarrollo y de valores para la vida. Se logra la sensibilización de estos agentes para comprender la diversidad como valor enriquecedor y positivo para todos, desde el respeto, la tolerancia, empatía y la igualdad de oportunidades, respetando básicamente su condición como ser humano, que garantice además de inclusión educativa y la plena inclusión en la sociedad.

PALABRAS CLAVE: discapacidad; inclusión educativa; inclusión social; necesidades educativas especiales.

\section{A LOOK AT INCLUSION AS AN OPPORTUNITY FOR DEVELOPMENT}

\section{ABSTRACT}

The investigation approaches experiences on the attention to people with special, associate educational necessities or not to discapacitys, from the relationship that settles down among the contexts scholar, family and community. It is developed in the municipality Bayamo through socialization and involvement workshops, in those that is considered as essential elements, the

\footnotetext{
${ }^{1}$ Licenciada en Educación. Especialidad Defectología. Máster en Dirección. Doctor en Ciencias Pedagógicas. Metodóloga de Posgrado Universidad de Granma, Cuba. Orcid: 0000-0001-6235-3221.lgarciagrillo@udg.co.cu

${ }^{2}$ Licenciada en Educación Especial. Máster en Ciencias de la Educación. Doctor en Ciencias Pedagógicas. Directora de Relaciones Interinstitucionales. Universidad de Granma. Cuba. Orcid: 0000-0002-2747-9627 yvigoae@udg.co.cu ${ }^{3}$ Licenciada en Educación Primaria. Máster en Ciencias de la Educación

${ }^{4}$ Hilda Brigitte Quijije Quiroz, Estudiante de la carrera Tecnologías de la Información en la Universidad Estatal del Sur de Manabí, Jipijapa - Manabí - Ecuador, Orcid: 0000-0002-6696-3075, quijije.hilda2868@unesum.edu.ec 
Lidia Esther García Grillo, Yisell Vigoa Escobedo, Dayamí Álvarez Ayala, Hilda Brigitte Quijije Quiroz

feedback that should exist enters the school, the family and the community like agents potenciadores of the development and values for life. The sensitization of these agents is possible to understand to the diversity like enriching and positive value for all, from the respect, the tolerance, empathy and the equality of opportunities, respecting its condition like human being that it guarantees besides its educational inclusion, basically its full inclusion in the society.

KEYWORDS: disability; educational inclusion; special educational necessities; social inclusión.

\section{INTRODUCCIÓN}

El abordaje al tema de la inclusión es una de las grandes preocupaciones que ha devenido en debate científico en diferentes contextos en los últimos años; donde la idea principal se orienta hacia la educación de las personas con necesidades educativas especiales asociadas o no a discapacidades y la determinación del contexto más favorecedor para su desarrollo.

Las autoras consideran que un aspecto medular en este sentido gira en torno al papel de la educación, y las influencias educativas de los contextos formativos para lograr el cambio de conciencia y de compromiso que se necesita para que la inclusión sea un hecho y se reconoce que la escuela, la familia y la comunidad, juegan un rol fundamental para lograr el mejoramiento humano, por ello es preciso su comprensión, como una relación necesaria, que requiere perfeccionamiento en el que todos tenemos participación y en esta juega un papel primordial la comunidad, por ser el contexto donde se ubican a la familia y a la escuela, y donde el sujeto, o sea el individuo, tiene acción y participación.

Pedagogos y psicólogos cubanos, coinciden en que es en la familia y la escuela, mediados por la sociedad, donde se inicia la socialización del ser humano. Estos contextos se distinguen por estar abiertos a las influencias recíprocas y cooperar entre sí. Las ideas planteadas demuestran la necesidad de la estrecha relación que debe existir entre estas instituciones sociales, donde los vínculos de jerarquía no deben predominar, pero sí los de coordinación e interrelación, para lograr que cada uno desde su posición contribuya de manera armónica a la formación y desarrollo integral del ser humano.

Con el objetivo de perfeccionar la atención a las personas con necesidades educativas especiales, asociadas o no a discapacidades, las autoras proponen talleres de socialización e inclusión, en los que se consideran como elementos esenciales, la retroalimentación que debe existir entre la escuela, la familia y la comunidad como agentes potenciadores del desarrollo.

\section{DESARROLLO}

En la actualidad, el término diversidad es muy polémico, genera varios puntos de vista y discusiones científicas. Se utiliza para referirse a circunstancias de la vida que se presentan de maneras diversas. Es un fenómeno característico del ser humano, a ello se une el término, atención a la diversidad, que ha ido evolucionando desde modelos segregadores a otros más inclusivos, sobretodo, desde que en el informe Warnock (1978) se definió el concepto de Necesidades Educativas Especiales (NEE).

96 UNESUM-Ciencias. Publicación cuatrimestral. Vol. 5, Año 2021, No. 6 (Especial Ingenierías) 
Se considera, que la diversidad, se encuentra presente en todas las actividades de la vida cotidiana y, por supuesto, también en la educación. Es considerada como algo propio de estos tiempos, resulta inherente a la sociedad y se puede percibir desde múltiples perspectivas: escolar, profesional, cultural, ideológica, ecológica, entre otras.

Para entender la inclusión, es preciso comprender que lo esencial de cada uno es su condición de ser humano, es decir es la diversidad que nos caracteriza, a decir de muchos "ser diferentes es algo común”, la diversidad es personal y cultural, es una característica inherente a la naturaleza humana y lo más importante es respetar a los demás, reconocer y valorar lo diverso que somos todas las personas, que tenemos culturas diferentes, que procedemos de contextos diferentes, de familias diferentes, interactuamos en contextos diferentes. Ha de servir además, para identificar a las personas como son, y no como nadie desearía que fueran y esto pudiera ser fácil comprenderlo, pero requiere de mucha atención y sensibilidad.

Es por ello, que en la educación, además de tener en cuenta la individualidad del hombre, como ser singular y único; es preciso tener en cuenta a la sociedad, como configuración cultural en la que se desenvuelve y desarrolla este hombre y en la que adquiere carácter social, donde las influencias del medio juegan un rol esencial en la formación y desarrollo del individuo, en la adaptación al medio y permite además su autotransformación y transformación, tanto en lo individual, como en lo social.

$\mathrm{Al}$ abordar la relación entre la escuela, la familia y la comunidad, es preciso tener en cuenta la vigencia que tiene la obra del filósofo Engels, F. (1884), al definir a la familia como la célula de la sociedad en su obra "La familia, la propiedad privada y el Estado". En la misma aporta una concepción innegable sobre el lugar que tiene la familia en la sociedad. Su fortaleza consistió en demostrar que la familia es una categoría histórica y social, no puede ser analizada al margen del contexto histórico y cultural en el cual se desarrolla. Este planteamiento fue una trascendental contribución en la comprensión del desarrollo social, sin duda la familia constituye un escenario de análisis importante, para entender el estado y las características por las que transita cualquier sociedad y el papel que esta juega en el proceso de inclusión de las personas con necesidades educativas especiales asociadas o no a discapacidades y también es evidente el papel que desempeña la sociedad en el desarrollo de cada familia, ya que esta se conforma y se configura en lo social, es el intermediario entre sociedad e individuo y a su vez es portadora de las normas sociales.

Por otra parte, López, R (2002) reconoce, que la escuela debe ser una institución abierta a la diversidad, que se responsabilice con garantizar educación de calidad para todos sus alumnos, a pesar de sus diferencias, pero, para ello, resulta preciso que el personal encargado de la organización, orientación y control del proceso, tenga la preparación necesaria y pueda ofrecer una respuesta acertada a cada situación que se le presenta en el contexto.

Por otra parte, Fernández, A (2011), plantea que el contexto comunitario es la agrupación de personas que se perciben como una unidad social, donde se evidencia una estructura organizativa, donde se comparte un territorio, intereses y necesidades, interactuando entre sí y promoviendo acciones colectivas a favor del crecimiento personal y colectivo, manifestando sentimientos de pertenencia como expresión de su identidad comunitaria. Esta autora reconoce la necesidad de la 
Lidia Esther García Grillo, Yisell Vigoa Escobedo, Dayamí Álvarez Ayala, Hilda Brigitte Quijije Quiroz

unidad de todos los miembros para lograr el crecimiento personal y colectivo, sin embargo, no ha sido suficientemente abordada la necesidad de saberes, recursos personológicos construidos que reflejan lo común, pero a su vez lo diverso de la comunidad.

Atendiendo a estos elementos, es preciso tener en cuenta que para lograr la inclusión, es preciso la relación armónica entre la escuela, la familia y la comunidad, por ello Borges S y Orozco M (2014), consideran que, la inclusión educativa es entendida como una concepción que reconoce el derecho de todos a una educación de calidad, independientemente de sus particularidades y características que condicionan las variabilidades en su desarrollo y que propicie su integración a la sociedad como individuos plenos en condiciones de poder disfrutar las posibilidades que ella ofrece y contribuir a su perfeccionamiento.

El logro de la inclusión demanda desafíos crecientes en la relación que se establece entre los diferentes contextos en que el individuo se desarrolla, y en la formación como seres humanos que los prepara para la vida, teniendo en cuenta además de los contextos; las transformaciones socio económicas y políticas que se producen en la sociedad actual y que promueve tanto el desarrollo espiritual como personal de cada individuo y su aporte al desarrollo local.

Es necesario, la comprensión y precisión de lo que le corresponde a cada cual en este proceso. La escuela, consciente de su importante función, perfecciona el proceso docente-educativo y labora para fortalecer la vinculación más estrecha con la familia y el entorno social donde está enclavada, en aras de cumplir con éxito su misión; debe saber determinar cuáles son las principales estrategias didáctico-metodológicas del maestro para dirigir el proceso de enseñanzaaprendizaje desarrollador de sus educandos, establecer una adecuada relación del aprendizaje con la vida como una dimensión de trabajo importante y potenciar los Pilares del aprendizaje para el siglo XXI determinados por la UNESCO 2001, aprender a ser, aprender a conocer, aprender a hacer, aprender a vivir juntos y aprender a emprender, recordando siempre que la ternura, el respeto y el amor, son complementos básicos en la labor educativa.

Si la educación, en estrecha relación con los diferentes contextos, dígase familia y comunidad, prepara a los educandos para la vida, para una vida activa, de derechos y deberes, de participación social plena, de justicia, de bienestar, de desarrollo, de éxito y de valores, logrará hacer realidad tanto la inclusión educativa como social de estas y los formará como mejores seres humanos, lo que favorece la igualdad de oportunidades y la participación de todos en el proyecto social de su localidad.

Tomando en cuenta lo referido anteriormente, las autoras proponen talleres de socialización para perfeccionar la atención a las personas con necesidades educativas especiales, asociadas o no a discapacidades, desde los tres contextos de actuación bajo las siguientes temáticas:

1. Inclusión educativa e inclusión social. Principales fundamentos.

2. Las personas con necesidades educativas especiales. Particularidades del desarrollo.

3. Las personas con necesidades educativas especiales. Proyecto de vida.

4. El papel de la familia, la escuela y comunidad en el desarrollo de las personas con necesidades educativas especiales asociadas o no a discapacidades.

5. La comunidad contexto favorecedor del desarrollo para las personas con necesidades educativas especiales.

98 UNESUM-Ciencias. Publicación cuatrimestral. Vol. 5, Año 2021, No. 6 (Especial Ingenierías) 
6. La escuela, la familia y la comunidad, entes activos para lograr la inclusión.

7. Proyectos de vida.

Las principales acciones desarrolladas fueron:

Determinar necesidades de superación para el contexto escolar, familiar y comunitario, sobre el conocimiento de las particularidades del desarrollo de las personas con necesidades educativas especiales, y la participación de la comunidad en la atención a estas personas y acciones de sensibilización a los factores de los tres contextos. Además, se realizó la observación directa a algunas actividades que realizan las personas con necesidades educativas especiales, en los contextos escuela, familia y comunidad, así como la revisión de varios documentos.

\section{Métodos}

Se manejó los métodos de investigación científica, en los que destacan histórico -lógico, inducción - deducción y análisis - síntesis, estos métodos permitieron examinar los antecedentes de la investigación, veracidad de la información, interpretación y contextualización de los contenidos abarcados para de esta forma poder obtener resultados que arriben a las conclusiones pertinentes de acuerdo a la búsqueda del conocimiento y aporte en la sociedad a la que se direcciona el trabajo de investigación así mismo los métodos bibliográficos, al conocer cada antecedente descrito en este trabajo.

\section{CONCLUSIONES}

Para el logro de la inclusión, se precisa del esfuerzo de todos, y de la armónica relación que se logre entre la escuela, la familia y la comunidad como contextos favorecedores de esta política educativa, y que debe lograrse desde un proceso activo, consciente y cooperativo, capaz de satisfacer las necesidades educativas de todos y cada uno de sus educandos, aprovechando las potencialidades y respetando lo diverso de cada individuo, actuando para su transformación como ser humano, donde la diversidad sea un valor enriquecedor y positivo para todos, desde el respeto a la diversidad, la igualdad de oportunidades que posibilite el desarrollo de cada una de estas personas y la creación y rediseño de los proyectos de vida.

Uno de los enfoques a considerar radica en la participación activa en el accionar de espacios de inclusión que fortalezcan el crecimiento personal y colectivo, que fomenten el desarrollo de habilidades y capacidades ludo-pedagógicas de los educandos, considerando como principal aspecto el rol significativo en la aplicación de derechos para la vida y la participación en la sociedad.

\section{REFERENCIAS BIBLIOGRÁFICAS}

Bell Rodríguez, R. y López Machín, R. (2002). Convocados por la diversidad. La Habana: Pueblo y Educación. Borges Rodríguez, S., Orosco Delgado, M. (2014). Inclusión educativa y Educación Especial: Un horizonte singular y diverso para igualar oportunidades de desarrollo. La Habana. Ed: Educación cubana.

Castellanos Simons, D. y otros. (2002). Aprender y enseñar en la escuela. La Habana: Pueblo y Educación.

Ferrari, M. (2004). La inclusión y sus desafíos en el siglo XXI. Educación, No. 112. (segunda época), mayo-agosto. 2004. La Habana. 
Lidia Esther García Grillo, Yisell Vigoa Escobedo, Dayamí Álvarez Ayala, Hilda Brigitte Quijije Quiroz

García Grillo, L., Virelles Espinosa, I. y Arceo Estrada, E. (2014, mayo). Familia y Educación. La Demajagua. p. 5. López Machín, R. (2006). Diversidad e igualdad de oportunidades en la escuela. La Habana: Pueblo y Educación.

López Machín, Ramón. Escolares con necesidades educativas especiales. Selección de temas. La Habana: Editorial Pueblo y Educación; 2011.

Warnock, H. M. Necesidades educativas especiales. “Informe del Comité de investigación sobre educación de niños y jóvenes deficientes “. Rev. Educación No. extraordinario. 1987. $152 \mathrm{p}$.

100 UNESUM-Ciencias. Publicación cuatrimestral. Vol. 5, Año 2021, No. 6 (Especial Ingenierías) 